\title{
Dewetting films: bifurcations and concentrations
}

\author{
A L Bertozzi ${ }^{1,2}$, G Grün $^{3}$ and T P Witelski ${ }^{1}$ \\ ${ }^{1}$ Department of Mathematics and Center for Nonlinear and Complex Systems, Duke University, \\ Durham, NC 27708-0320, USA \\ 2 Department of Physics, Duke University, Durham, NC 27708-0305, USA \\ ${ }^{3}$ Institut für Angewandte Mathematik, Universität Bonn, Beringstr. 6, 53115 Bonn, Germany
}

Received 10 August 2000, in final form 3 May 2001

Published 21 September 2001

Online at stacks.iop.org/Non/14/1569

Recommended by F Otto

\begin{abstract}
Under the influence of long-range attractive and short-range repulsive forces, thin liquid films rupture and form complex dewetting patterns. This paper studies this phenomenon in one space dimension within the framework of fourth-order degenerate parabolic equations of lubrication type. We derive the global structure of the bifurcation diagram for steady-state solutions. A stability analysis of the solution branches and numerical simulations suggest coarsening occurs. Furthermore, we study the behaviour of solutions in the limit that shortrange repulsive forces are neglected. Both asymptotic analysis and numerical experiments show that this limit can concentrate mass in $\delta$-distributions.
\end{abstract}

Mathematics Subject Classification: 34B15, 35G25, 35K55, 35Q35

\section{Introduction}

The competition of attractive van der Waals forces and short-range repulsive forces, such as Born repulsion, can produce complex instabilities in layers of thin liquid films on solid substrates. Experimental studies have shown this behaviour for polymer films [33,50,54, 55, 63], liquid crystal films [28,57,58], liquid metals [6,28] and evaporating films [13,45]. During these dewetting processes, large droplets are formed which are connected by ultra-thin films. In analogy to spinodal decomposition in phase separation for binary alloys described by the Cahn-Hilliard equation, this evolution is sometimes called spinodal dewetting [42, 43, 45].

The lubrication approximation describes the motion of long-wave unstable thin films of thickness $u$ by a fourth-order degenerate parabolic partial differential equation (PDE) of the form $[10,47]$

$$
\frac{\partial u}{\partial t}-\nabla \cdot(M(u) \nabla p)=0
$$


where the pressure $p$ in the thin film is given by

$$
p=P(u)-\Delta u \text {. }
$$

The explicit form of the nonlinear mobility $M(u)$ depends on the boundary condition at the liquid-solid interface. The forms

$$
M(u)=u^{3} \quad \text { and } \quad M(u)=u^{3}+\beta u^{b}
$$

with $\beta>0, b \in(0,3)$, correspond to no-slip and various classes of slip boundary conditions, respectively. The Laplacian term in (2) gives the linearized contribution of surface tension to the total pressure. The term $P(u)$ encompasses forces exerted on the film, such as a hydrostatic body force and disjoining/conjoining intermolecular forces due to van der Waals interactions and Born repulsion [15]. This latter effect is the focus of this paper; we examine a class of pressure relations $P(u)$ describing the competition of disjoining and conjoining pressure components of singular type. Generically, we consider functions

$$
P(u)=\frac{1}{u^{n}}-\frac{\epsilon^{m-n}}{u^{m}}=\frac{1}{u^{n}}\left(1-\left[\frac{\epsilon}{u}\right]^{m-n}\right) \quad 0<n<m
$$

where $\epsilon$ is a small positive parameter-as considered in the context of partial wetting films [14].

The standard 6-12 Lennard-Jones potential [30,42,43,47] corresponds to the exponents $(n, m)=(3,9)$ in this model. The $u^{-n}$ term describes the aforementioned long-range attractive force, while the second term in (4) models the short-range stabilizing effect of Born repulsion. Oron and Bankoff $[45,46]$ consider $(4)$ with $(n, m)=(3,4)$ to describe a thin film on a layered solid substrate. Our results show that the solutions of these models have the same qualitative structure for a large set of $(n, m)$ values.

Early studies of van der Waals-driven instabilities of thin films, by Williams and Davis $[7,60]$ and de Gennes [10], considered this problem without repulsive pressure terms, i.e. $P(u)$ given by equation (4) with $\epsilon=0$. In general, classical solutions of this problem can cease to exist in finite time due to singularities which occur if the film ruptures, $u \rightarrow 0$. In this context, for $n=3$, self-similar finite-time rupture solutions were studied by Zhang and Lister [64] and Witelski and Bernoff [61,62].

In contrast, for $\epsilon>0$, the problem is globally well-posed, and solutions exist for all times. In [27], Grün and Rumpf studied the convergence of numerical schemes suggested in [26]. As a by-product, existence of strictly positive, unique and smooth solutions to equations (1) and (2) was proved-provided the dimension is $d=1$ and $m>3$. Corresponding results for multiple space dimensions can be found in [25]. Numerical simulations of thin films with generalized van der Waals disjoining pressure show complex pattern formation during which films evolve to a metastable state composed of a collection of droplets connected by a thin film of thickness approximately $\epsilon$. A comparison with physical experiments shows striking similarities to the characteristic features of dewetting including the formation of capillarity ridges at the edges of growing holes (cf [27]).

We show that the parameter $\epsilon$ in (4) gives a minimum thickness for non-trivial thin-film equilibrium states. Viewed as a regularization, it is natural to consider the limit of these 'postrupture' solutions as $\epsilon \rightarrow 0$. We show via numerical simulations and asymptotic analysis of the equilibria, that concentrations [16] occur in this model. That is, this limit makes sense only as a measure; for fixed times after rupture numerical simulations show the $\epsilon \rightarrow 0$ limit concentrate mass as a $\delta$-distribution.

Appropriate boundary conditions for equation (1) corresponding to the physical problem of a fluid confined in an impermeable solid container are

$$
\boldsymbol{n} \cdot \nabla p=0 \quad \boldsymbol{n} \cdot \nabla u=0 \quad \text { on } \quad \partial \Omega .
$$


The latter condition specifies a $90^{\circ}$ contact angle between the fluid surface and vertical boundaries at $\partial \Omega$. This condition makes a uniform fluid layer, $u(x, y)=\bar{u}$, an exact solution of the full problem and is physically equivalent to neglecting any positive or negative meniscus due to wetting or non-wetting properties of the container holding the fluid. By the former condition, mass is conserved, as is the average film thickness $\bar{u}$ which is given by

$$
\bar{u}=\frac{1}{\mathcal{A}} \int_{\Omega} u \mathrm{~d} A
$$

where $\mathcal{A}=\operatorname{area}(\Omega)$. We use $\bar{u}$ as a bifurcation parameter for the set of equilibrium solutions of (1). An energy, or Lyapunov integral, for (1) is given by

$$
\mathcal{E}=\int_{\Omega} \frac{1}{2}|\nabla u|^{2}+Q(u) \mathrm{d} A
$$

where $Q$ is the primitive of $P$,

$$
Q(u)=-\int_{u}^{\infty} P(v) \mathrm{d} v .
$$

This energy is monotonically decreasing, with the rate of dissipation given by

$$
\frac{\mathrm{d} \mathcal{E}}{\mathrm{d} t}=-\int_{\Omega} M(u)|\nabla p|^{2} \mathrm{~d} A \leqslant 0 .
$$

In the next section we prove that in one dimension the local minimizers of the energy correspond to states with a constant uniform pressure, $p=\bar{p}$, and that they solve the semilinear elliptic equation

$$
\bar{p}=P(u)-\Delta u \quad \text { on } \Omega
$$

with Neumann boundary conditions. Solutions satisfy the compatibility condition

$$
\bar{p}=\frac{1}{\mathcal{A}} \int_{\Omega} P(u) \mathrm{d} A .
$$

In this paper, we confine ourselves to the case of two-dimensional thin films, i.e. $\Omega$ is given as anterval $(0, L)$ and $u$ solves the equation

$$
\frac{\partial u}{\partial t}+\frac{\partial}{\partial x}\left(M(u) \frac{\partial}{\partial x}\left[\frac{\partial^{2} u}{\partial x^{2}}-P(u)\right]\right)=0 .
$$

This is a special case of the general long-wave unstable model [5,36-38]

$$
u_{t}=-\left(F(u) u_{x x x}\right)_{x}-\left(G(u) u_{x}\right)_{x}
$$

with $F(u)=M(u)$ and $G(u)=-P^{\prime}(u) M(u)$. In section 2 we prove the existence of a positive global minimizer of the energy (7) with mass constraint (6) in one dimension. In section 3 we rigorously derive the complete structure of the set of equilibrium solutions in one dimension. We show that all non-trivial steady states lie on branches that bifurcate from trivial solutions. In section 4 we compute the local bifurcation structure near critical values on the branch of constant solutions. In section 5 we study stability of the equilibria, showing that the only non-trivial steady states are those that lie on the first bifurcating branch. In section 6 we investigate the asymptotic structure of the steady solutions in the limit as $\epsilon \rightarrow 0$. For a fixed mass, the non-trivial stable solution concentrates in a $\delta$-distribution. This behaviour is also observed in results from simulations of the dynamics for a sequence of time-dependent solutions (see figure 9). 


\section{Positive solutions of the PDE and minimizers in one space dimension}

In this section we discuss the existence theory for the one-dimensional problem and prove the existence of a smooth global minimizer of the Lyapunov functional (7). For a pressure of the form (4), we have the following theorem for positive initial data:

Theorem 1. Consider initial data satisfying

$u_{0}>0 \quad u_{0} \in H^{1}(\Omega) \quad$ and $\quad \frac{1}{2} \int_{\Omega}\left|\partial_{x} u_{0}\right|^{2} \mathrm{~d} x+\int_{\Omega} Q\left(u_{0}\right) \mathrm{d} x<\infty$

and assume that $0<n<m, m \geqslant 3$ in (4). Then a unique positive smooth solution of (1)-(4) exists for all $t>0$.

Proof. Following arguments from previous papers [4,5] it suffices to derive a priori pointwise upper and lower bounds for the solution. We derive an a priori bound on the $H^{1}$ norm and show this implies pointwise bounds. Then uniform parabolicity implies that the solution is completely smooth. Similar arguments are presented in [4] for the case $P(u)=0$ and in [5] for a destabilizing non-singular $P(u)$.

First we note that the Lyapunov functional implies that for any time $T>0$,

$$
\frac{1}{2} \int_{\Omega}\left|\partial_{x} u(T)\right|^{2} \mathrm{~d} x \leqslant \frac{1}{2} \int_{\Omega}\left|\partial_{x} u_{0}\right|^{2} \mathrm{~d} x+\int_{\Omega} Q\left(u_{0}\right) \mathrm{d} x-\int_{\Omega} Q(u(T)) \mathrm{d} x .
$$

The initial data are in $H^{1}$ and positive. Since $m>n,-Q(u)$ has an a priori upper bound independent of $u$, implying an a priori bound for $\int\left|\partial_{x} u\right|^{2} \mathrm{~d} x$ at the later time $T$. In one space dimension this immediately implies an a priori pointwise upper bound on $u$ and also on the $C^{1 / 2}$ norm, at time $T$.

To show that $u$ has a pointwise a priori lower bound at time $T$ note that the above implies $\int Q(u(T)) \mathrm{d} x \leqslant C$. Let $u_{\min }=\min _{\Omega} u$ be attained at $x_{0}$. By Hölder continuity, $u(x) \leqslant u_{\min }+C_{h}\left|x-x_{0}\right|^{1 / 2}$, where $C_{h}$ is the Hölder constant. Hence

$C>\int Q(u) \mathrm{d} x \geqslant C(\epsilon) \int\left(u_{\min }+C_{h}\left|x-x_{0}\right|^{1 / 2}\right)^{-m+1} \mathrm{~d} x+\mathrm{O}(1) \geqslant C(\epsilon) \eta\left(u_{\min }\right)+\mathrm{O}(1)$

where $\eta\left(u_{\min }\right)=-\log u_{\min }$ for $m=3$ and $\eta\left(u_{\min }\right)=u_{\min }^{3-m}$ for $m>3$. Thus the solution is forbidden to go below a positive threshold for $m \geqslant 3$. Note that all the bounds depend strongly on $\epsilon$ as it nears zero. In particular, these results are not true for the case $\epsilon=0$.

Remark. In higher space dimensions or when $P(\cdot)$ does not contain singular repulsive terms, strict positivity of corresponding solutions cannot be guaranteed. Hence, the classical Schauder approach used in the one-dimensional setting has to be replaced by an ansatz involving weaker solution concepts $[9,24,25]$. Moreover, the positivity condition on initial data might be replaced by the weaker condition that initial data are non-negative and that the total energy is initially bounded.

The long-time behaviour of solutions of the PDE is related to minimizers of the Lyapunov functional (7). Define $Q(u)$ to be $\infty$ for $u \leqslant 0$; we can then prove the following results concerning the global minimizer. By direct methods of the calculus of variations, we deduce the following theorem in arbitrary space dimensions.

Theorem 2. Let $\Omega$ be a bounded domain of class $C^{0,1}$, let $\bar{u}>0$ and assume $0<n<m$ in (4). Then a global minimizer of $\mathcal{E}(\cdot)$ (7) exists in the class $V:=\left\{u \in H^{1}(\Omega): \int_{\Omega} u \mathrm{~d} x=\bar{u}\right\}$. In particular, $u>0$ almost everywhere in $\Omega$. 
Proof. Let $\left(u_{k}\right)_{k \in N}$ be a minimizing sequence which clearly exists since $\mathcal{E}\left(u_{0}\right)<\infty$ for $u_{0} \equiv \bar{u}$. Observe that $Q(\cdot)$ is bounded from below by a constant $B \in \mathbb{R}$. Hence, a constant $C$ exists such that $\int_{\Omega}\left|\nabla u_{k}\right|^{2} \mathrm{~d} x \leqslant C<\infty$ for all $k \in N$.

Rellich's compactness result implies that a subsequence (again denoted by $\left(u_{k}\right)_{k \in N}$ ) exists which converges strongly in $L^{2}(\Omega)$ and pointwise almost everywhere to a function $u \in H^{1}(\Omega)$. By Fatou's lemma, we deduce that also $Q(u)$ lies in $L^{1}(\Omega)$. Using the weak lower semicontinuity of the norm, we obtain

$$
\mathcal{E}(u) \leqslant \liminf _{k \rightarrow \infty} \mathcal{E}\left(u_{k}\right)=\inf _{w \in V} \mathcal{E}(w) .
$$

Consequently, $u$ is a minimizer and the integrability of $Q(u)$ implies that $u>0$ almost everywhere in $\Omega$.

Corollary 1. In space dimension $d=1, u$ is strictly positive on $\Omega$, provided $m \geqslant 3$ in (4).

The proof of this result is identical to the positivity of solutions of the evolution equation (see theorem 1). If $m \geqslant 3$ and $d=1$, the minimizer constructed above is sufficiently regular in order to give a meaning to the corresponding Euler-Lagrange equations. This allows us not only to obtain better regularity results for solutions, but also to investigate their qualitative properties as solutions of a second-order ordinary differential equation (ODE).

Theorem 3. Let $m \geqslant 3$ and $0<n<m$ in (4). Then a minimizer $u$ as constructed in theorem 2 is a solution of the ODE on $\Omega=\{x \mid 0 \leqslant x \leqslant L\}$,

$$
\begin{aligned}
& P(u)-u_{x x}=\bar{p} \\
& u_{x}(0)=u_{x}(L)=0 \quad \frac{1}{L} \int_{0}^{L} u \mathrm{~d} x=\bar{u} .
\end{aligned}
$$

Moreover, u is smooth.

Proof. Due to the strict positivity of $u$, the function $F_{\phi}(s):=\mathcal{E}(u+s \phi), \phi \in H^{1}(\Omega)$ is differentiable for $s$ in $(-\delta, \delta)$ for sufficiently small $\delta$. Since $u$ is a minimizer, by differentiation of $F_{\phi}(s)$ at $s=0$, we obtain

$$
\int_{0}^{L} u_{x} \phi_{x} \mathrm{~d} x+\int_{0}^{L} P(u) \phi \mathrm{d} x=0 \quad \forall \phi \in H^{1}(\Omega) \quad \int_{0}^{L} \phi \mathrm{d} x=0 .
$$

Here, $P(u)$, given by (4), is continuous due to the strict positivity of $u$. Rewriting this for arbitrary $\Psi \in H^{1}(\Omega)$ yields

$$
\int_{0}^{L} u_{x} \Psi_{x} \mathrm{~d} x+\int_{0}^{L} P(u) \Psi \mathrm{d} x=\int_{0}^{L} \bar{p} \Psi \mathrm{d} x \quad \forall \Psi \in H^{1}(\Omega)
$$

where $\bar{p}$ is given by (11). Observing that $P(u)-\bar{p}$ is of class $C^{\alpha}$, elliptic regularity theory [23] ensures that $u$ is a strong solution, i.e. of class $C^{2}$ with vanishing normal derivatives at the boundary. In particular, the right-hand side is of class $C^{2}$. We may iterate, and the $C^{\infty}$-property for $u$ follows. 


\section{One-dimensional steady-state solutions}

From theorem 3 we know that all minimizers of $\mathcal{E}$ are smooth steady-state solutions on $0 \leqslant x \leqslant L$ satisfying

$$
\begin{aligned}
& P(u)-u_{x x}=\bar{p} \\
& u_{x}(0)=u_{x}(L)=0 \quad \bar{p}=\frac{1}{L} \int_{0}^{L} P(u) \mathrm{d} x .
\end{aligned}
$$

In this section we examine the number and structure of steady-state solutions.

While aspects of this problem parallel the Cahn-Hilliard and constrained Allen-Cahn equations $[1,2,12,22,44]$, the nonlinear function $P(u)$ in (17) has a different form from that considered in most previous works and leads to a different structure of the bifurcation diagram.

Equation (17) can be written as an autonomous phase plane system,

$$
u_{x}=v \quad v_{x}=P(u)-\bar{p}
$$

where $\bar{p}$ is a control parameter. Observe that every Neumann solution of (17) and (18) on $x \in(0, L)$ can be extended by reflection symmetry to yield a periodic solution of (19) on $x \in(0,2 L)$. Hence, a necessary condition for the existence of Neumann solutions is that (19) admits periodic solutions. Moreover, the symmetry of (19) guarantees that periodic solutions on $[0,2 L]$ can be shifted to yield Neumann solutions on $[0, L]$.

Let $P_{\max }$ be the maximum of $P(u)$ on $u>0$. Observe that for any $P(u)$ of the form (4) and $\bar{p} \in\left(0, P_{\max }\right)$, equation (19) has a unique elliptic centre point at $u_{c}$ satisfying $P\left(u_{c}\right)-\bar{p}=0$ with $P^{\prime}\left(u_{c}\right)<0$. Note that for $\bar{p}<0$ there are no elliptic equilibria, and hence no periodic orbits and no Neumann solutions of (17). In addition, for $0 \leqslant \bar{p}<P_{\max }$, equation (19) has a hyperbolic saddle point at $u_{s} \sim \epsilon+\epsilon^{n+1} \bar{p} /(m-n)$ as $\epsilon \rightarrow 0$ with $u_{c}>u_{s}$. The stable and unstable manifolds of the saddle point form a homoclinic orbit that encloses $u_{c}$ and all of the periodic solutions of (19). Periodic solutions surrounding the centre $u_{c}$ have a minimum greater than the saddle-point value $u_{s}$. Therefore, every non-trivial equilibrium solution is bounded from below by $u_{s}, u(x)>\epsilon$.

Equation (18) has the trivial uniform constant solution $u=\bar{u}$ for any positive value $\bar{u}>0$; the corresponding average pressure is $\bar{p}=P(\bar{u})$. Branches of non-trivial solutions can bifurcate from the constant solutions only at singular points $\bar{u}_{*}$, where the linear operator $\mathcal{L}=P^{\prime}\left(\bar{u}_{*}\right)-\partial_{x x}$ has a non-trivial nullspace [31]; hence the bifurcation points are given by

$$
P^{\prime}\left(\bar{u}_{k}\right)=-k^{2} \pi^{2} / L^{2} \quad k=1,2, \ldots, K
$$

For $\epsilon>0, P^{\prime}(u)$ is bounded from below for $u>0$, therefore there are only a finite number of branches, $k=1,2, \ldots, K$. For each $k$, there are two roots, $\bar{u}_{k}^{+}>\bar{u}_{k}^{-}$, with $P^{\prime \prime}\left(\bar{u}_{k}^{+}\right)>0$ and $P^{\prime \prime}\left(\bar{u}_{k}^{-}\right)<0$. It is straightforward to show that as $\epsilon \rightarrow 0, K=\mathrm{O}\left(\epsilon^{-(n+1) / 2}\right)$ by noting that the minimum scales like $P^{\prime}\left(u^{*}\right)=\mathrm{O}\left(\epsilon^{-(n+1)}\right)$, where the critical point scales as $u^{*}=\mathrm{O}(\epsilon)$. For $\epsilon=0$ there are an infinite number of upper solutions $\bar{u}_{k}^{+}$while the lower solutions $\bar{u}_{k}^{-}$ disappear.

In this section we consider $\epsilon>0$ and prove that for each $k$ there is a branch of nontrivial steady-state solutions that bifurcates from $\bar{u}_{k}^{+}$and reconnects at $\bar{u}_{k}^{-}$. We consider an interval of fixed length $L$ and examine the bifurcating branches of solutions as the total mass, $L \bar{u}=\int_{0}^{L} u(x) \mathrm{d} x$, varies. We begin by specifying the range of $m$ and $n$ for which our results hold. 


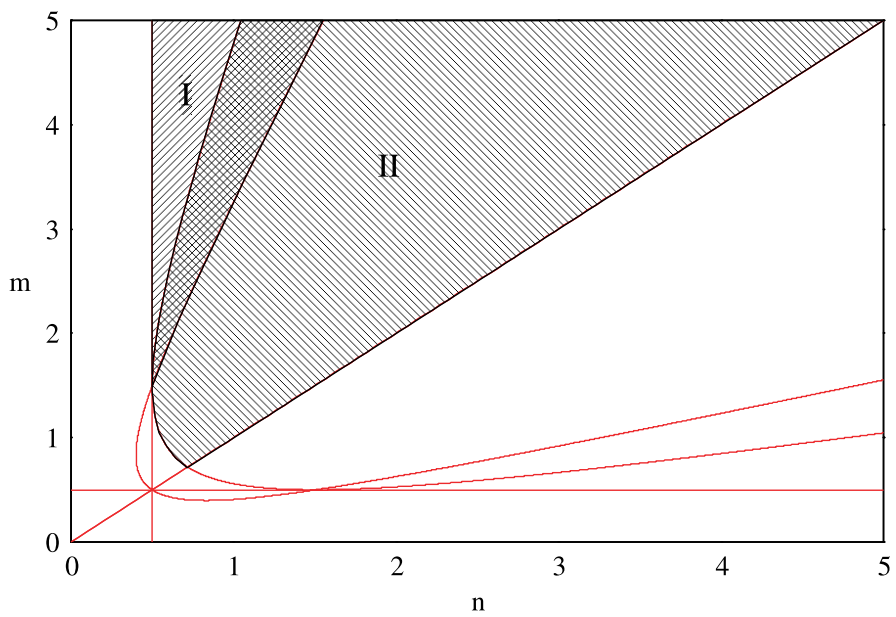

Figure 1. The set of admissible values for $(n, m)$ satisfying definition 1 (shaded regions for cases I and II).

Definition 1. We call a pair of positive exponents $(n, m)$ in (4) admissible if they satisfy $m>n$ and either (I) or (II):

$$
\begin{aligned}
& \left\{n>\frac{1}{2} \text { and } 3 n^{2}-10 m n+3 m^{2}-m-n+2>0\right\} \\
& \left\{3 n^{2}-14 m n+3 m^{2}-2 m-2 n+7<0\right\} .
\end{aligned}
$$

A diagram of the set of admissible values given by definition 1 is shown in figure 1 .

The main result of this section is the following theorem.

Theorem 4. Consider $P(u)$ as given in (4) and admissible $(n, m)$ in the sense of definition 1 . Then, for each positive integer $k \leqslant K$ there exists a non-trivial branch of solutions to (17) and (18). Parametrized by $\bar{p} \in\left(P\left(\bar{u}_{k}^{+}\right), P\left(\bar{u}_{k}^{-}\right)\right)$, each branch of non-trivial solutions bifurcates from $\bar{u}_{k}^{+}$and reconnects to the constant state branch at $\bar{u}_{k}^{-}$. Furthermore, all solutions of (17) and (18) are either trivial constant solutions or lie on one of these bifurcating branches.

Figure 2 shows the bifurcation diagram for the set of equilibria of (17) and (18). At each $\bar{u}$, the diagram gives the maximum and minimum values of each solution $u(x)$. Specifying two out of three of the values $u_{\min }, u_{\max }, \bar{u}$, specifies an equilibrium solution $u(x)$, unique up to the reflection symmetry, $x \rightarrow L-x$.

Theorem 4 is proved in lemmas 1 and 3 below.

Lemma 1. For $\bar{p} \in\left(0, P_{\max }\right)$, parametrize the family of periodic solutions $u(x)$ of (19) by their minimum,

$$
q=\min _{x} u(x) .
$$

Furthermore, let $L_{k}(q)$ be the length of the $x$-interval on which $u(x)-\bar{u}$ has exactly $k$ zeros and $u(x)$ satisfies the Neumann boundary conditions (18). Then for each $k, L_{k}$ is a monotonically decreasing function of $q$ and $L_{k}(q) \rightarrow \infty$ for each $k$ as $q \searrow u_{s}$.

Proof. This lemma is a direct consequence of a general result by Schaaf [52], which can be summarized as follows. 


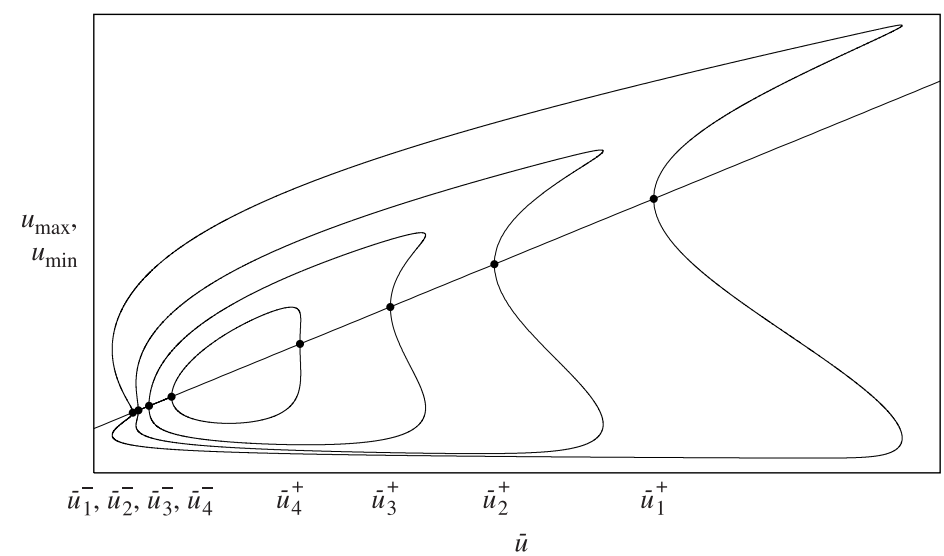

Figure 2. Bifurcation diagram of equilibria for (4) with $(n, m)=(3,4)$ and $\epsilon=0.14$, yielding $K=4$ branches.

Proposition 1. Consider the two-point boundary value problem

$$
u_{x x}+f(u)=0 \quad u_{x}(0)=u_{x}(L)=0 .
$$

Assume $f\left(u_{0}\right)=0, f^{\prime}\left(u_{0}\right)>0$, and $f$ has only one other root at $u_{1}<u_{0}$. Suppose that $f$ is strictly negative in a right neighbourhood of $u_{1}$ and that it is continuous in $u_{1}$. If in addition $S:=f^{\prime} f^{\prime \prime \prime}-\frac{5}{3}\left(f^{\prime \prime}\right)^{2}<0$ for all $u \geqslant u_{1}$, then there is a family of solutions parametrized by $u_{1}<q<u_{0}$ with corresponding interval length $L_{k}(q)$ ( $q$ and $L_{k}$ are as defined in lemma 1$)$ such that for all $k \geqslant 1, L_{k}(q)$ is a monotonically decreasing function of $q$.

Proof. This proposition is a direct consequence of several results stated in Schaaf [52]. In particular, by definition 1.4.1 of [52] $f$ is an ' $A$-function'. In addition, proposition 1.5.6 of [52] says that if $f$ is an $A$-function, $f^{\prime}<0$ near $u_{1}, f<0$ near $u_{1}$ and $\lim _{u \rightarrow u_{1}} f(u)=0$ then $f$ is an $A-B$ function. This implies (theorems 1.4.2 and 2.1.3 of [52]) that $L_{k}^{\prime \prime}(q)>0$ for all $u_{1}<q<u_{0}, i \in Z^{+}$. Also $L_{k}^{\prime}\left(u_{0}\right)=0$ so that $L_{k}$ is strictly decreasing. Finally, note that the phase portrait has nested solutions inside a homoclinic orbit, thus the period approaches infinity as $q \rightarrow u_{s}$.

Lemma 2. Let $P(u)$ be as defined in (4). Let $\epsilon>0$ and assume that $(n, m)$ in (4) are admissible in the sense of definition 1. Then $f(u)=\bar{p}-P(u)$ is an A-function on $\mathbb{R}^{+}$, i.e. $S(u)=P^{\prime} P^{\prime \prime \prime}-\frac{5}{3}\left(P^{\prime \prime}\right)^{2}<0$ on $\mathbb{R}^{+}$.

Proof. For $P(u)$ given by (4), the Schaaf functional $S(u)$ can be written in the form

$$
S(u)=\frac{N(w)}{3 u^{2 n+4}} \quad w=(\epsilon / u)^{m-n}>0
$$

where

$$
\begin{gathered}
N(w):=m^{2}(m+1)(1-2 m) w^{2}-m n\left(3 n^{2}-10 m n+3 m^{2}-m-n+2\right) w \\
+n^{2}(n+1)(1-2 n) .
\end{gathered}
$$

Note that $S(u)$ is the ratio of a quadratic polynomial in $w, N(w)$, over a positive-definite function of $u$. Therefore, to show that $S(u)<0$ for $u>0$ it is sufficient to guarantee that 
the quadratic polynomial is negative. First, $N(w)$ must be concave, hence $m>\frac{1}{2}$. Then there are two cases; (I) the maximum of $N(w)$ occurs for $w<0$ and (II) the maximum occurs for $w>0$. In case I, to satisfy the $A$-function condition, it is sufficient to show that $N(0) \leqslant 0$, yielding $n>\frac{1}{2}$, and that $N^{\prime}(0)<0$,

$$
3 n^{2}-10 m n+3 m^{2}-m-n+2>0 .
$$

This equation describes the region outside a branch of a rotated hyperbola in the first quadrant of the $(n, m)$-plane (see figure 1$)$. To show that $S(u)<0$ in case II, it is sufficient to show that the maximum of $N(w)$ is negative. Following some algebraic manipulations, this condition reduces to

$$
3 n^{2}-14 m n+3 m^{2}-2 m-2 n+7<0
$$

describing the interior of a different rotated hyperbola (see figure 1). Figure 1 shows the set of admissible $(n, m)$ subject to all of the above constraints as shaded regions for cases I and II.

Lemma 1 implies the following characterization of the bifurcation diagram.

Lemma 3. Let $P$ be as defined in (4) with $m$ and $n$ satisfying definition 1 and let $k$ be a positive integer less than or equal to $K$. Then the following hold.

(a) For all $\bar{p} \in\left(P\left(\bar{u}_{k}^{+}\right), P\left(\bar{u}_{k}^{-}\right)\right)$, there exists a non-trivial solution $u_{k}=u_{k}(\cdot, \bar{p})$ of $(17)$ and (18) such that $u_{k}-\bar{u}_{k}$ has precisely $k$ zeros.

(b) Modulo reflections $x \rightarrow L-x$, this solution is unique.

(c) The non-trivial solution $u_{k}(\cdot, \bar{p})$ depends continuously on $\bar{p}$ for $\bar{p} \in\left[P\left(\bar{u}_{k}^{+}\right), P\left(\bar{u}_{k}^{-}\right)\right]$. As $\bar{p} \rightarrow P\left(\bar{u}_{k}^{ \pm}\right), u_{k}(\cdot, \bar{p})$ approaches the constant solutions $\bar{u}_{k}^{ \pm}$.

(d) All non-trivial steady-state solutions of (17) lie on one of the solution branches $u_{k}(x, \bar{p})$, with $P\left(\bar{u}_{k}^{+}\right) \leqslant \bar{p} \leqslant P\left(\bar{u}_{k}^{-}\right)$.

Remark. For each $k$, the family $u_{k}(\cdot, \bar{p})$, with $\bar{p} \in\left[P\left(\bar{u}_{k}^{+}\right), P\left(\bar{u}_{k}^{-}\right)\right]$, defines a branch of solutions that bifurcates from the constant states. Each of these branches originates at a constant value $\bar{u}_{k}^{ \pm}$where an eigenvalue of the linearized problem changes sign. In the next section we perform weakly nonlinear asymptotic analysis that shows that the bifurcations are generically either subcritical or supercritical pitchfork bifurcations.

Proof of lemma 3. Recall that the length of the interval $L$ is fixed and let the period map $L_{k}(q, \bar{p})$ be as introduced in lemma 1 . Now we emphasize the dependence of $L_{k}$ on $\bar{p}$ explicitly. Recall that for fixed $\bar{p}, L_{k}(q, \bar{p})$ tends monotonically to $k \pi / \sqrt{\left|P^{\prime}\left(u_{c}\right)\right|}$ as $q$ approaches the elliptic centre point $u_{c}=u_{c}(\bar{p})$. Furthermore, $L_{k}(q, \bar{p})$ tends monotonically to infinity when $q$ decreases to the hyperbolic saddle point $u_{s}=u_{s}(\bar{p})$. In the proof of proposition 1 (see [52]), we found that for each $\bar{p}, L_{k}^{\prime \prime}(q)>0$ for $u_{s}<q \leqslant u_{c}$, and $L_{k}^{\prime}\left(u_{c}\right)=0$. Moreover, this period map is a smooth function of $q$ on $\left(u_{s}, u_{c}\right]$. Therefore, $L_{k}$ is a monotonically decreasing function on this interval and defines a bijection

$$
L_{k}(\cdot ; \bar{p}):\left(u_{s}, u_{c}\right] \rightarrow\left[\frac{k \pi}{\sqrt{\left|P^{\prime}\left(u_{c}\right)\right|}}, \infty\right)
$$

also see figure 3 for a graph of $L_{1}(q, \bar{p})$. Thus existence of a non-trivial solution $u_{k}$ occurs when $L$ is in the range of $L_{k}(q, \bar{p})$. Therefore, to prove (a), it is sufficient to show that

$$
\bar{p} \in\left(P\left(\bar{u}_{k}^{+}\right), P\left(\bar{u}_{k}^{-}\right)\right) \quad \Rightarrow \quad L \in\left(\frac{k \pi}{\sqrt{\left|P^{\prime}\left(u_{c}\right)\right|}}, \infty\right)
$$




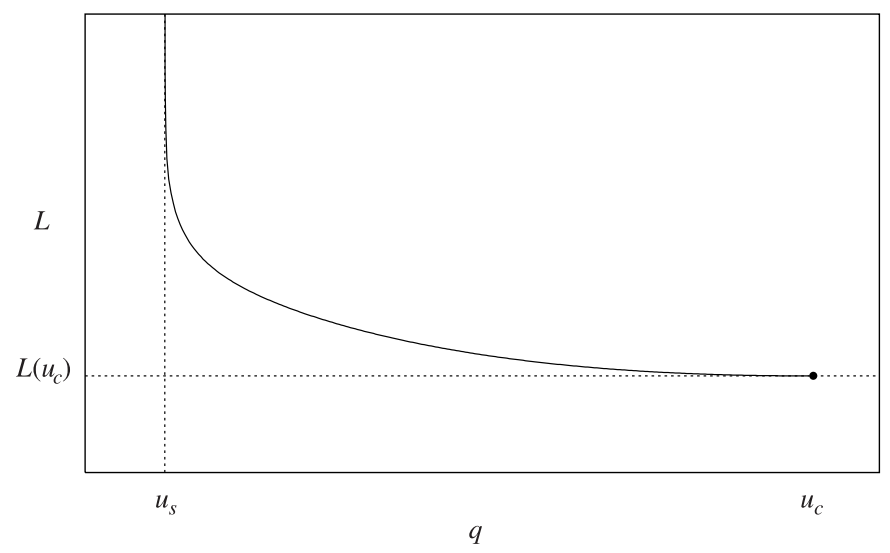

Figure 3. $L_{1}(q ; \bar{p})$ for $\bar{p}=5, \epsilon=0.2$.

where $\bar{u}_{k}^{ \pm}$are given by (20). From direct calculation with (4) for $\bar{p} \in\left(P\left(\bar{u}_{k}^{-}\right), P\left(\bar{u}_{k}^{+}\right)\right)$, i.e. for $u_{c} \in\left(\bar{u}_{k}^{-}, \bar{u}_{k}^{+}\right), P(\cdot)$ satisfies

$$
\left|P^{\prime}\left(u_{c}(\bar{p})\right)\right|>\left|P^{\prime}\left(\bar{u}_{k}^{ \pm}\right)\right|=\frac{k^{2} \pi^{2}}{L^{2}}
$$

and we use the fact that $P\left(u_{c}(\bar{p})\right)=\bar{p}$. This implies that $L$ is in the range of $L_{k}(\cdot, \bar{p})$,

$$
L>\frac{k \pi}{\sqrt{\left|P^{\prime}\left(u_{c}(\bar{p})\right)\right|}}
$$

and hence this proves (a).

Conversely for $\bar{p} \notin\left[P\left(\bar{u}_{k}^{-}\right), P\left(\bar{u}_{k}^{+}\right)\right], L_{k}(q)$ is always greater than $L$ so there are no nontrivial solutions. This proves (d). Assertion (b) follows from the Picard-Lindelöf theorem.

To finish the proof, we prove continuity of the bifurcating branches of solutions, $u_{k}(\bar{p})$, as a function of the parameter $\bar{p}$. Note that solutions of the differential equation (17) depend continuously on the parameter $\bar{p}$ and on initial conditions $u_{x}=0, u=q$. Thus, it remains to be shown that $q(\bar{p}) \equiv \min _{x} u_{k}(x ; \bar{p})$ depends continuously on $\bar{p}$. To show this we apply the implicit function theorem to the map $W(q, \bar{p})=L_{k}(q, \bar{p})-L$. Note that all non-trivial equilibria correspond to solutions of $W(q(\bar{p}), \bar{p})=0$. From the proof of (a) we know that for all $\bar{p} \in\left(P\left(\bar{u}_{k}^{-}\right), P\left(\bar{u}_{k}^{+}\right)\right)$, the Jacobian $D W$ is invertible with respect to the first argument. Hence $q(\bar{p})$ depends continuously on $\bar{p}$ for all $\bar{p} \in\left(P\left(\bar{u}_{k}^{-}\right), P\left(\bar{u}_{k}^{+}\right)\right)$.

The final step is to prove the limiting result for the endpoints of the interval. From continuity of $L_{k}(q, \bar{p})$ and $u_{c}(\bar{p})$, for $\bar{p} \rightarrow P\left(\bar{u}_{k}^{ \pm}\right)$,

$$
L_{k}\left(u_{c}\left(P\left(\bar{u}_{k}^{ \pm}\right)\right), P\left(\bar{u}_{k}^{ \pm}\right)\right)=\frac{k \pi}{\sqrt{\left|P^{\prime}\left(u_{c}\left(P\left(\bar{u}_{k}^{ \pm}\right)\right)\right)\right|}} \quad u_{c}\left(P\left(\bar{u}_{k}^{ \pm}\right)\right)=\bar{u}_{k}^{ \pm} .
$$

Thus,

$$
\lim _{\bar{p} \rightarrow P\left(\bar{u}_{k}^{ \pm}\right)} L_{k}\left(u_{c}(\bar{p}), \bar{p}\right)=\frac{k \pi}{\sqrt{\left|P^{\prime}\left(\bar{u}_{k}^{ \pm}\right)\right|}}=L .
$$

Recall that $L_{k}(\cdot, \bar{p})$ is continuously invertible on $\left(u_{s}(\bar{p}), u_{c}(\bar{p})\right]$. Hence,

$$
\min _{x} u_{k}\left(x, P\left(\bar{u}_{k}^{ \pm}\right)\right)=q_{k}\left(P\left(\bar{u}_{k}^{ \pm}\right)\right)=L_{k}^{-1}(L)=u_{c}\left(P\left(\bar{u}_{k}^{ \pm}\right)\right)=\bar{u}_{k}^{ \pm}
$$


i.e. the solution $u_{k}\left(\cdot, P\left(\bar{u}_{k}^{ \pm}\right)\right)$is identical to the constant solution $\bar{u}_{k}^{ \pm}$by virtue of PicardLindelöf's theorem. This proves the lemma.

We note that the index $k$ is also called the lap number $[3,22]$.

\section{Local structure of the equilibrium bifurcation diagram}

We analytically compute the local structure of equilibrium bifurcation points using perturbation methods [29, 32]; we use a strained parameter expansion to calculate the local bifurcation diagram from the Lyapunov-Schmidt reduction $[12,20]$. For simplicity we assume $L=1$. Let $0 \leqslant \sigma \ll 1$ be an expansion parameter for non-trivial solutions near a bifurcation point $\bar{u}_{*}$,

$$
u(x)=\bar{u}_{*}+\sigma u_{1}(x)+\sigma^{2} u_{2}(x)+\sigma^{3} u_{3}(x)+\cdots .
$$

For convenience, we write the average pressure, $\bar{p}$, as $P(u)$ applied to an effective uniform film thickness, $U(\sigma)$, that also depends on $\sigma$,

$$
\bar{p}=P(U(\sigma)) \quad U(\sigma)=U_{0}+\sigma U_{1}+\sigma^{2} U_{2}+\sigma^{3} U_{3}+\cdots .
$$

Substituting these expansions into (17) yields a regular perturbation problem as $\sigma \rightarrow 0$. At leading order, $P\left(\bar{u}_{*}\right)=P\left(U_{0}\right)$ and hence $U_{0}$ is one of the bifurcation values $\bar{u}_{k}$, $k=1,2,3, \ldots, K$. At $\mathrm{O}(\sigma)$, we obtain the linear equation

$$
\hat{\mathcal{L}} u_{1} \equiv P^{\prime}\left(\bar{u}_{k}\right) u_{1}-u_{1 x x}=P^{\prime}\left(\bar{u}_{k}\right) U_{1}
$$

with the solution $u_{1}(x)=A \cos (k \pi x)+U_{1}$ for $P^{\prime}\left(\bar{u}_{k}\right)=-k^{2} \pi^{2}$, see equation (20), and $A$ is the amplitude of the solution. At next order, we obtain

$$
\hat{\mathcal{L}} u_{2}=-P^{\prime \prime}\left(\bar{u}_{k}\right)\left[A U_{1} \cos (k \pi x)+\frac{1}{2} A^{2} \cos ^{2}(k \pi x)\right] .
$$

Applying the Fredholm alternative to ensure that the right-hand side is in the range space of the linear operator $\hat{\mathcal{L}}$ determines a unique solution $u_{2}(x)$ subject to the condition that $U_{1}=0$. This observation reduces (27) to the form (18) noted earlier. Finally, at $\mathrm{O}\left(\sigma^{3}\right)$, we use the equation for $u_{3}(x), \hat{\mathcal{L}} u_{3}=\mathcal{R}_{3}\left(\bar{u}_{k}, u_{1}, u_{2}, U_{3}\right)$, to determine $A$, the amplitude of the solution, where $\mathcal{R}_{3}$ is an inhomogeneous term. Applying the Fredholm alternative to this equation, $\int_{0}^{1} \mathcal{R}_{3} \cos (k \pi x) \mathrm{d} x=0$, determines the amplitude $A$. The condition that $A$ is real determines the sign of $U_{2}$. Apart from this condition, there are no other restrictions on $U_{2}$ that cannot be eliminated by a rescaling of variables. Hence, without loss of generality, we take $U(\sigma)=\bar{u}_{k} \pm \sigma^{2}$ where the sign is chosen appropriately to obtain a real solution,

$$
A_{k}=2 k \pi \sqrt{\left|\frac{6 P^{\prime \prime}\left(\bar{u}_{k}\right)}{5 P^{\prime \prime}\left(\bar{u}_{k}\right)^{2}+3 k^{2} \pi^{2} P^{\prime \prime \prime}\left(\bar{u}_{k}\right)}\right|} .
$$

Consequently, the average and extrema of the solution can be written as

$$
\begin{aligned}
& \bar{u}=\int_{0}^{1} u(x) \mathrm{d} x \sim \bar{u}_{k}-\sigma^{2} \frac{\operatorname{sgn}\left(P^{\prime \prime}\left(\bar{u}_{k}\right)\right)\left[3 k^{2} \pi^{2} P^{\prime \prime \prime}\left(\bar{u}_{k}\right)-P^{\prime \prime}\left(\bar{u}_{k}\right)^{2}\right]}{\left|5 P^{\prime \prime}\left(\bar{u}_{k}\right)^{2}+3 k^{2} \pi^{2} P^{\prime \prime \prime}\left(\bar{u}_{k}\right)\right|} \\
& u_{k, \min } \sim \bar{u}_{k}-\sigma A_{k} \quad u_{k, \max } \sim \bar{u}_{k}+\sigma A_{k} .
\end{aligned}
$$

Combining these results we obtain the local structure of the bifurcation near the bifurcation point $\bar{u}_{k}^{ \pm}$for $k=1,2, \ldots, K$,

$$
\left[u(0)-\bar{u}_{k}\right]^{2}=\frac{24 k^{2} \pi^{2} P^{\prime \prime}\left(\bar{u}_{k}\right)}{P^{\prime \prime}\left(\bar{u}_{k}\right)^{2}-3 k^{2} \pi^{2} P^{\prime \prime \prime}\left(\bar{u}_{k}\right)}\left(\bar{u}-\bar{u}_{k}\right) \geqslant 0 .
$$

We see that, except for isolated degenerate cases, all bifurcations are either supercritical or subcritical pitchfork bifurcations [15]. Figure 4 shows the bifurcation diagram for $P(u)$ given by (4) with $n=3, m=4$ and $\epsilon=0.18$ compared with (32). 


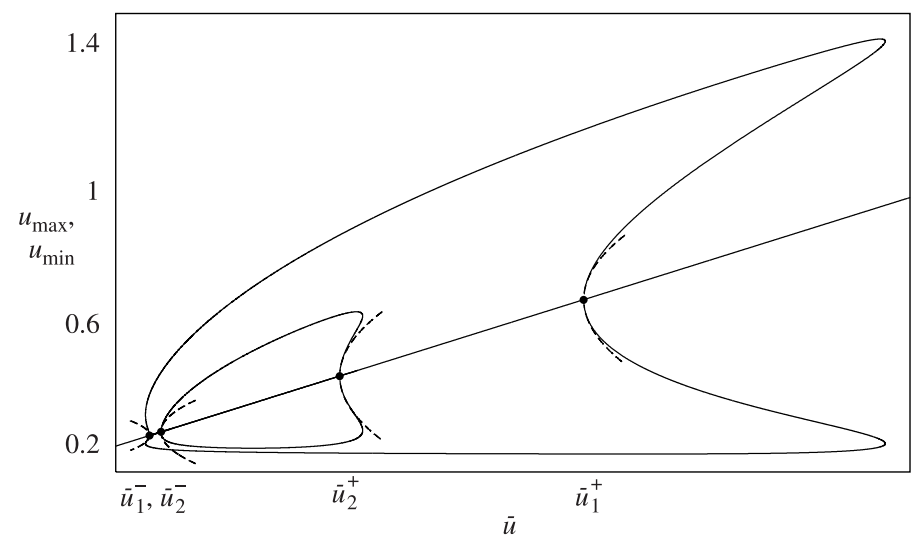

Figure 4. Numerical calculation of the bifurcation diagram for $P(u)=u^{-3}-\epsilon u^{-4}$ with $\epsilon=0.18$. Dashed curves show the analytic results for the local bifurcation structure (29)-(31).

\section{Stability of the equilibrium solutions}

In this section we consider the stability of equilibria with respect to mass-preserving perturbations. We begin with the linear analysis for uniform constant equilibrium solutions and go on to make more global statements for the entire set of equilibrium solutions.

If the initial data for the PDE (12), with $M(u), P(u)$ given by (3) and (4) is near an equilibrium $\underline{u}(x)$, then it is convenient to write the solution in the form $u(x, t)=\underline{u}(x)+\tilde{u}(x, t)$, where $\tilde{u}$ is a zero-mean perturbation. Linearizing (12) yields the evolution equation for $\tilde{u}$,

$$
\frac{\partial \tilde{u}}{\partial t}=\mathcal{L} \tilde{u} \equiv \partial_{x}\left(M(\underline{u}) \partial_{x}\left[P^{\prime}(\underline{u})-\partial_{x x}\right]\right) \tilde{u}
$$

with the corresponding linearized Neumann boundary conditions (5),

$$
\partial_{x x x} \tilde{u}=0 \quad \partial_{x} \tilde{u}=0 \quad \text { at } \quad x=0,1 .
$$

The choice $\tilde{u}(x, t)=\hat{u}(x) \mathrm{e}^{\lambda t}$ in (33) yields the associated Neumann eigenvalue problem, $\mathcal{L} \hat{u}=\lambda \hat{u}$.

We note that the linear stability of the uniform constant solutions $\underline{u}(x)=\bar{u}$ can be computed directly [62]. For these states

$$
\lambda_{\ell}=-M(\bar{u}) \ell^{2} \pi^{2}\left(P^{\prime}(\bar{u})-\ell^{2} \pi^{2}\right) \quad \ell=1,2,3, \ldots
$$

with corresponding eigenmodes $\hat{u}=A \cos (\pi \ell x)$.

We now briefly summarize the results of a standard weakly nonlinear analysis near the bifurcation points. In an $\varepsilon$ neighbourhood of the bifurcation point $\bar{u}_{k}, \bar{u}=\bar{u}_{k}+\varepsilon$, the eigenvalues are $\lambda_{k, \ell} \sim-\ell^{2} \pi^{2} M\left(\bar{u}_{k}\right)\left(P^{\prime}\left(\bar{u}_{k}\right)-\ell^{2} \pi^{2}+\varepsilon P^{\prime \prime}\left(\bar{u}_{k}\right)\right)$. From (20), the eigenvalue $\lambda_{k, k}=\mathrm{O}(\varepsilon)$ and crosses zero at the bifurcation point. Combining the dominant eigenvalue of the trivial solution with the local bifurcation structure found in section 4 yields the amplitude equation for the dynamics near the first bifurcation point,

$$
\frac{\mathrm{d} A}{\mathrm{~d} t} \sim-\pi^{2} M\left(\bar{u}_{1}\right) P^{\prime \prime}\left(\bar{u}_{1}\right) A\left(\frac{P^{\prime \prime}\left(\bar{u}_{1}\right)^{2}-3 \pi^{2} P^{\prime \prime \prime}\left(\bar{u}_{1}\right)}{24 \pi^{2} P^{\prime \prime}\left(\bar{u}_{1}\right)}-A^{2}\right)
$$

where $\bar{u}_{1}=\bar{u}_{1}^{ \pm}$. An equivalent amplitude equation can be written for the other bifurcation points $\bar{u}_{k}$; it describes weakly nonlinear dynamics of solutions restricted to the $k$-fold symmetry of the $k$ th branch of equilibrium solutions. 


\subsection{General linear stability theory for long-wave unstable lubrication equations}

The linear stability of long-wave unstable nonlinear equations of the type (12) has been considered recently for lubrication-type equations [36-38] and for a number of years for Cahn-Hilliard problems $[1-3,12,22,44]$. Some of the results from these papers are relevant for our problem and we briefly review them here.

The operator (33) is the composition of two second-order operators, $\mathcal{L} \hat{u}=\overline{\mathcal{M}} \overline{\mathcal{L}} \hat{u}$, where the mobility operator, $\overline{\mathcal{M}}$, and non-local operator, $\overline{\mathcal{L}}$, are, respectively

$$
\overline{\mathcal{M}} v \equiv \partial_{x}\left(M(\underline{u}) \partial_{x} v\right) \quad \overline{\mathcal{L}} w \equiv P^{\prime}(\underline{u}) w-w_{x x}-\frac{1}{L} \int_{0}^{L} P^{\prime}(\underline{u}) w \mathrm{~d} x
$$

for zero-mean $w(x)$ satisfying Neumann boundary conditions. We note that $\mathcal{L}$ is self-adjoint with respect to a weighted $H^{-1}$ norm using an inner product defined by the mobility. For every zero-mean function $w(x)$, there exists a unique zero-mean potential, $W$, such that $\overline{\mathcal{M}} W=w$. The weighted $H^{-1}$ inner product of two zero-mean functions $w$ and $v$ is then $\langle w, v\rangle \equiv \int M(\underline{u}) \partial_{x} W \partial_{x} V \mathrm{~d} x$. This norm can be used to describe the thin-film evolution as a gradient flow in $H^{-1}$. In this context, let us also mention the paper [48] by Otto which shows that the surface-tension-driven thin-film flow in the Hele-Shaw cell has a gradient flow structure with respect to the Wasserstein metric (see also [18] for a rigorous derivation of the lubrication approximation within that framework). As the operator $\mathcal{L}$ is self-adjoint and the domain is compact, the spectrum is real and discrete $[17,38]$. We note that related results on the properties of the linearized spectrum can be obtained from analysis of a Rayleigh quotient, as considered by Bates and Fife for the Cahn-Hilliard equation [3] and by Laugesen and Pugh $[36,38]$ for equations of the type (13).

In the case of constant mobility $M(u)=M$, Bai et al [1] and Grinfeld and NovickCohen [22] showed that the properties of the spectrum of (33) can be obtained from analysis of the second-order problem for $\overline{\mathcal{L}}$. Since the equilibria are smooth and bounded away from zero, the problem with non-constant mobility $M(u)$ can be treated equivalently; the operator $\overline{\mathcal{M}}$ is invertible and hence $\mathcal{L} \hat{u}=\lambda \hat{u}$ can be written as $\overline{\mathcal{L}} \hat{u}=\lambda \overline{\mathcal{M}}^{-1} \hat{u}$. These results directly extend to (33) using the homotopy

$$
\overline{\mathcal{L}} \hat{u}=\lambda\left[\alpha+(1-\alpha) \overline{\mathcal{M}}^{-1}\right] \hat{u}
$$

where $\alpha=0$ gives the fourth-order operator $\mathcal{L}$ and $\alpha=1$ gives the second-order operator $\overline{\mathcal{L}}$. Lemma 1.2 in [22] and theorem 3.1 in [1] show that the number of positive and negative eigenvalues of (38) is independent of $0 \leqslant \alpha \leqslant 1$. Furthermore, Grinfeld and Novick-Cohen's theorem 5.1 [22] for excluding $\lambda=0$ from the spectrum of $\mathcal{L}$ directly extends to our problem and can be stated as

Theorem 5. Consider the linearized equation (33) with $M(u), P(u)$ given by (3) and (4). Then, away from the bifurcation points $\bar{u}_{k}^{ \pm}$on the line of constant solutions, and apart from turning points, $\lambda=0$ is not in the spectrum of (33) on the branches of non-trivial solutions. No secondary bifurcations are possible.

The proof follows directly from [22] with appropriate changes for $M(u)$ and $P(u)$.

\subsection{Existence of stable non-trivial solutions}

For the Neumann problem for (13), Laugesen and Pugh have proved that equilibrium solutions with interior critical points are unstable (see theorem 4 [38]). We now combine these results to prove the existence of a family of stable non-trivial solutions for (12). 
Theorem 6. For problem (12) with $P(u)(4)$ satisfying theorem 4 and $M(u)$ given by (3), there exists an interval $I=\left(\bar{u}_{1}^{\min }, \bar{u}_{1}^{\max }\right)$ containing $\left(\bar{u}_{1}^{-}, \bar{u}_{1}^{+}\right)$such that for each $\bar{u} \in I$, there exists a stable non-trivial steady-state solution. These stable monotonic solutions lie on the first branch of solutions, $k=1$.

Proof. Theorems 2 and 3 ensure the existence of a globally stable solution that minimizes the energy (7) under the mass constraint (6). The linear stability results (35) show that the uniform constant solutions are unstable for $\bar{u} \in\left(\bar{u}_{1}^{-}, \bar{u}_{1}^{+}\right)$. Likewise, theorem 4 of Laugesen and Pugh [38] shows that the non-trivial solutions branches with $k$-fold symmetry for $k=2,3, \ldots, K$ must also be unstable. Therefore, stable solutions for $\bar{u}_{1}^{-}<\bar{u}<\bar{u}_{1}^{+}$can only exist as part of the $k=1$ branch of non-trivial monotonic solutions. By theorem 5 the stability of any portion of a branch of solutions extends up to turning points. Then, there exist turning points $\bar{u}=\left\{\bar{u}_{1}^{\min }, \bar{u}_{1}^{\max }\right\}$ that define the largest interval containing $\left(\bar{u}_{1}^{-}, \bar{u}_{1}^{+}\right)$for which there are stable solutions on the first branch.

Remark. Figures 2 and 3 with $\epsilon=0.14$ and 0.18 , respectively, show that for moderately small $\epsilon$ the branches have a very simple structure. There is precisely one turning point for each subcritical bifurcation. We conjecture that this is generically true for this problem with $P(u)$ of the form (4). In this case, we can determine the number of positive eigenvalues for all solutions along the first bifurcating branch. In figure 3, the first branch has two unstable portions, one near each of the bifurcation points. By theorem 6 , on the portion of the non-trivial branch between the turning points, all of the solutions must be stable. In the next section we study the asymptotic structure of this branch in the limit as $\epsilon \rightarrow 0$.

Corollary 2. For $k=2, \ldots, K$, a subset of solutions along the kth branch of non-trivial solutions (connecting $u_{k}^{-}$to $u_{k}^{+}$) is stable with respect to perturbations with the same $k$-fold symmetry.

This result follows directly from theorem 6 by noting that the $k$ th bifurcating branch for the problem with domain size $L$ is the first bifurcating branch for the problem with domain size $L / k$. Instability of these reduced-domain-stable solutions to perturbations on the full domain is called coarsening [3]. An example of this effect can be seen in the numerical simulation described in figure 10 of the final section of this paper.

\section{Asymptotic structure of equilibrium solutions for $\epsilon \rightarrow 0$}

We now study the structure of the equilibrium solutions of (17) and (18) in the limit that $\epsilon \rightarrow 0$. Our approach in this section is a leading-order matched asymptotic analysis [32] of the solution branches. Figure 5 shows the first branch for $\epsilon=0.1$ with $(n, m)=(3,4)$. This branch has subcritical pitchfork bifurcations at $\bar{u}_{1}^{+}$and $\bar{u}_{1}^{-}$. Turning points occur on this branch at $\bar{u}_{1}^{\max }$ and $\bar{u}_{1}^{\mathrm{min}}$, respectively, describing the maximum and minimum average film thicknesses for non-trivial solutions. As described above, the turning points separate families of stable and unstable solutions on this branch. The unstable solutions have a finite-amplitude structure, with $u_{\min }=\mathrm{O}(1)$ as $\epsilon \rightarrow 0$, while the stable solutions are localized droplets connected by an ultra-thin film with thickness $u \sim \epsilon$ (see figure 6) and approach the homoclinic solution of (19). We show that these stable droplet solutions concentrate mass as a $\delta$-distribution in the limit of vanishing $\epsilon$. Moreover, from the asymptotic analysis, we obtain an estimate for the turning point $\bar{u}_{1}^{\max }$ as a function of $\epsilon$. 


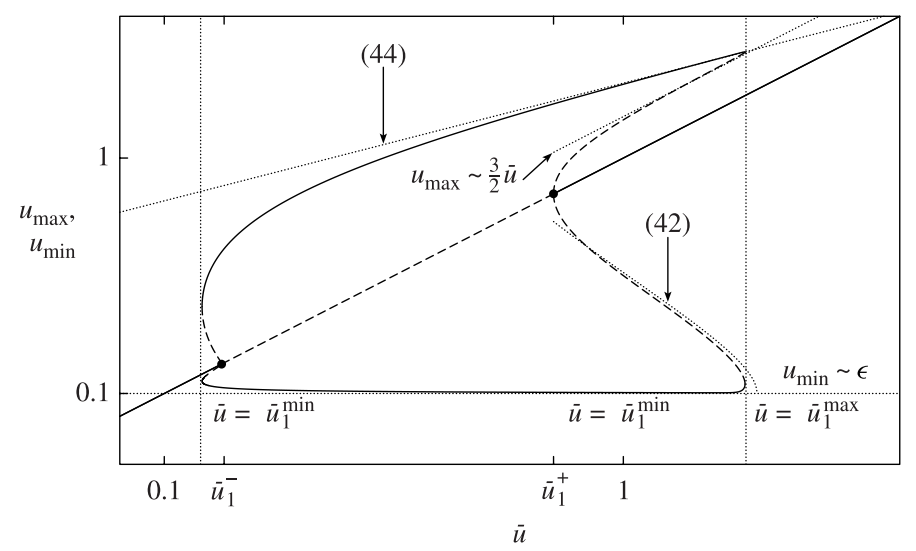

Figure 5. Log-log plot of the asymptotic structure for the first branch of solutions in the bifurcation diagram for $\epsilon=0.1$.

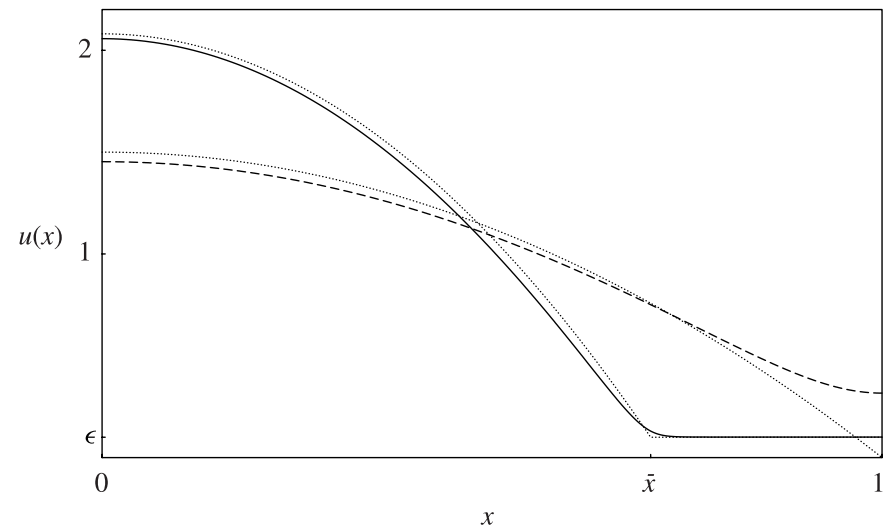

Figure 6. The stable (full) and unstable (broken) equilibrium solutions, and the leading-order asymptotic approximations (dotted), for $\epsilon=0.1$ and $(n, m)=(3,4)$ with $\bar{u}=1$, and the leadingorder asymptotic approximations (dotted).

Recall the equation for equilibria (17) on $0 \leqslant x \leqslant 1$,

$$
P(u)-u_{x x}=\bar{p} \quad \bar{p}=\int_{0}^{1} P(u) \mathrm{d} x .
$$

Uniform and weakly non-uniform solutions of (39) are accurately described by the weakly nonlinear bifurcation analysis of section 4 . Weakly nonlinear solutions, with $u(x) \sim \bar{u}=\mathrm{O}(1)$ as $\epsilon \rightarrow 0$, have $\bar{p} \sim P(\bar{u})$, hence the local and average pressure terms in (39) are of comparable size everywhere. In contrast, for large amplitude solutions, we can simplify equation (39) in regions where $\bar{p} \gg P(u)$. Let $P_{0}(u) \equiv u^{-n}$ denote the pressure function $P(u)$ in (4) with $\epsilon=0$. For $\epsilon=0$ we note that $\bar{p} \geqslant P_{0}(\bar{u})$ is a consequence of Jensen's inequality. For $0<\epsilon \ll 1$, we can obtain asymptotic estimates for the branches of solutions away from the bifurcation points.

We focus on the $k=1$ branch of non-trivial solutions. Without loss of generality, we consider monotonically decreasing solutions with the maximum of the solution at $x=0$ and the minimum at $x=1$ (see figure 6). We will describe the structure of these solutions, 
parametrized by $\bar{u}$, in the asymptotic limit $\epsilon \rightarrow 0$. By rescaling variables, the asymptotic structure of the first branch also applies to the other solution branches, $k=2,3, \ldots, K$ (see the remark below).

Neglecting the local pressure $P(u)$, reduces (39) to the problem $-u_{x x}=\bar{p}$ with the parabolic droplet solution

$$
u(x) \sim \frac{1}{2} \bar{p}\left(\bar{x}^{2}-x^{2}\right) \quad 0 \leqslant x<\bar{x}
$$

with the maximum

$$
u_{\max }=u(0) \sim \frac{1}{2} \bar{p} \bar{x}^{2} .
$$

This is a leading-order outer solution for $\bar{p} \gg P(u)$. This outer solution does not hold as $x \rightarrow \bar{x}$, where (40) yields $u \rightarrow 0$ and the local pressure $P(u)$ makes a strong contribution. There, a rescaled inner problem must be solved to balance the curvature with the local pressure. The form of this problem depends on the size of $u_{\text {min }}$ compared with $\mathrm{O}(\epsilon)$. If $P(u)$ is locally greater than $\bar{p}$, then $P(u)$ must have a local maximum, with $\mathrm{d} P / \mathrm{d} x=P^{\prime}(u) u_{x}=0$. The maximum can occur either at the edges of the domain, where $u_{x}=0$, or at a point where $u(x)=u_{p}$, the unique root of $P^{\prime}\left(u_{p}\right)=0$, that is $u_{p}=(m / n)^{1 /[m-n]} \epsilon>\epsilon>0$. We now describe these two cases for the $k=1$ branch of solutions.

\subsection{Finite-amplitude solutions}

If $u_{\min }=\mathrm{O}(1) \gg \epsilon$, then $P(u) \sim P_{0}(u)$ everywhere. Furthermore, since $u_{\min }>u_{p}$, the maximum of $P(u)$ must occur at a boundary and we obtain a localized boundary layer correction at $\bar{x}=1$ (see figure 6 ). In this case, the leading-order average value of the solution is given by the integral of (40) on $0 \leqslant x \leqslant 1$, that is $\bar{u} \sim \bar{p} / 3$. Consequently, $\bar{p} \sim 3 \bar{u}$, and we obtain $u_{\max } \sim 3 \bar{u} / 2$ from (41). The corresponding values of $u_{\min }$ for these solutions can be found by solving an algebraic equation given by the first integral of (39),

$$
Q\left(u_{\max }\right)-Q\left(u_{\min }\right)=\bar{p}\left(u_{\max }-u_{\min }\right)
$$

where for $n>1$,

$$
Q(u)=-\frac{1}{u^{n-1}}\left(\frac{1}{n-1}-\frac{1}{m-1}\left[\frac{\epsilon}{u}\right]^{m-n}\right)
$$

to obtain $u_{\min }$ in terms of $\bar{u}$. These approximations for $u_{\max }$ and $u_{\min }$ for $\epsilon=0.1$ are shown in figure 5; these results describe the large- $\bar{u}$ portion of the unstable part of the $k=1$ solution branch. Comparison of these leading-order asymptotic approximations with the numerically calculated bifurcation diagram show negligible differences for $\epsilon \approx 0.05$ or smaller. As noted above, the structure of this part of the bifurcation diagram is independent of $\epsilon$ as $\epsilon \rightarrow 0$ and will converge to the results for $\epsilon=0$ given in [39,62].

\subsection{Localized parabolic droplet solutions}

The solutions considered above have pressure $P(u)$ strictly less than the maximum possible pressure $P_{\max }=P\left(u_{p}\right)=\mathrm{O}\left(\epsilon^{-n}\right)$. In contrast, if $u_{\min }<u_{p}=\mathrm{O}(\epsilon)$ then the monotonically decreasing solution must have an interior layer since the maximum of the pressure deviation, $P(u)-\bar{p}$, occurs within the domain, in a neighbourhood of the point $\bar{x},(40)$.

For these solutions, $\bar{p} \ll P_{\max }$, that is $P(u)=\mathrm{O}\left(\epsilon^{-n}\right)$ only on a small neighbourhood of $\bar{x}$ (see figure 7), so that the contribution of the maximum pressure to the average pressure, (39), is smaller than $\mathrm{O}\left(\epsilon^{-n}\right)$. Consequently, the matched asymptotic [32] description of these solutions consists of three parts: 


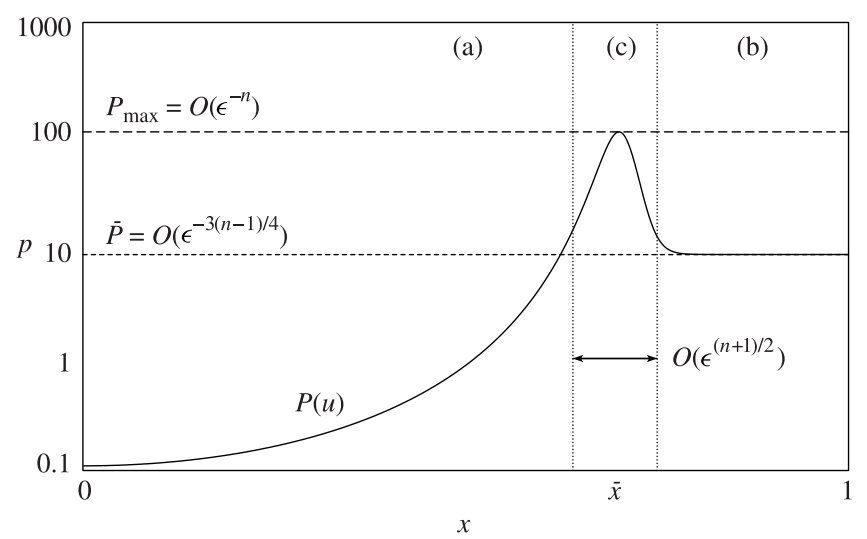

Figure 7. A linear-log plot of the local pressure $P(u)$ for the stable equilibrium solution $u(x)$ for $\epsilon=0.1$ with $\bar{u}=1$ (see figure 6). The three regimes for the matched asymptotic construction of the solution are shown: (a) the droplet, (b) the ultra-thin film and (c) the interior layer.

(a) the droplet, the outer solution (40) on $[0, \bar{x})$, where $u(x)$ and $P(u)$ are both $\mathrm{O}(1)$,

(b) the ultra-thin film, the outer solution describing the nearly flat solution on $(\bar{x}, 1]$ where $u=\mathrm{o}(1)$ and the local pressure is large,

(c) the interior layer, the inner solution in a small neighbourhood of $\bar{x}$ where $P(u)=\mathrm{O}\left(\epsilon^{-n}\right)$ and the curvature of $u(x)$ is large.

We begin with the structure of the ultra-thin film; there the solution $u(x)$ and its gradients are small. As a result, the leading-order balance in (39) is between the local and average pressures, $P(u) \sim \bar{p}$, with $u<u_{p}=\mathrm{O}(\epsilon)$. The condition that $\bar{p} \ll \mathrm{O}\left(\epsilon^{-n}\right)$ determines the constant value for the thickness of the ultra-thin film, $u_{\min }=u \sim \epsilon+\epsilon^{n+1} \bar{p} /(m-n)$. Note that the leading-order result, $u \sim \epsilon$, is given by the balance of attractive and repulsive terms in (4), $P(u)=0$. This value for $u_{\min }$ differs from the value of the saddle point $u_{s}$, found in section 3 , only by higher-order terms. Consequently, the structure of these equilibria is very similar to the homoclinic orbit of (19).

In the interior layer, the leading-order balance is between the second derivative and the local pressure, $u_{x x} \sim P(u)$, with $u=\mathrm{O}(\epsilon)$. This balance requires large gradients in $u(x)$ and determines the layer to be an $\mathrm{O}\left(\epsilon^{(n+1) / 2}\right)$ neighbourhood of $\bar{x}$. This result also shows that the local contribution of the pressure maximum to the average pressure, $\mathrm{O}\left(\epsilon^{1-n / 2}\right)$, is much smaller than $\mathrm{O}\left(\epsilon^{-n}\right)$, satisfying our initial assumption. Further details of the structure of the interior layer are not needed to complete the construction of the leading-order solution, as shown below.

Returning to (40), we can now complete the description of these solutions. Since $Q\left(u_{\max }\right) \ll Q(\epsilon)$, then from (42) we find that $u_{\max } \sim-Q(\epsilon) / \bar{p}$. From (41) we determine that $\bar{x} \sim \sqrt{2|Q(\epsilon)|} / \bar{p}$. Finally, integrating (40), we obtain the average value to leading order, $\bar{u} \sim(2|Q(\epsilon)|)^{3 / 2} /\left(3 \bar{p}^{2}\right)$, yielding the maximum value of the solution for this portion of the $k=1$ branch (see figure 5) as

$$
u_{\max } \sim\left(\frac{9|Q(\epsilon)|}{8}\right)^{1 / 4} \sqrt{\bar{u}}
$$

and the average pressure as

$$
\bar{p} \sim\left(\frac{2|Q(\epsilon)|}{3^{2 / 3}}\right)^{3 / 4} \frac{1}{\sqrt{\bar{u}}} .
$$




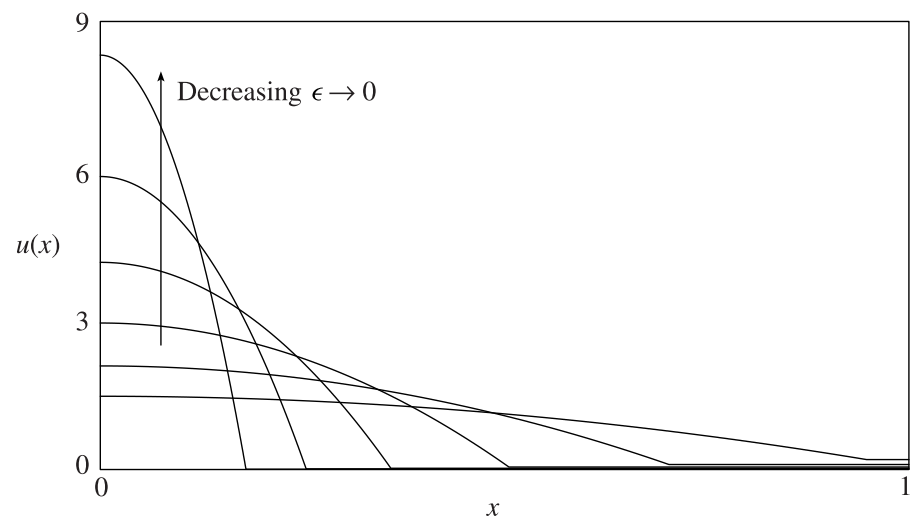

Figure 8. Stable solutions on the first branch for fixed mass $\bar{u}=1$ and values of $\epsilon=0.2,0.1$, $0.05,0.025,0.0125,0.00625$. The solutions become more concentrated at $x=0$ as $\epsilon$ decreases.

Consequently, as $\epsilon \rightarrow 0$, for each allowable fixed value of $\bar{u} \in\left(\bar{u}_{1}^{\min }, \bar{u}_{1}^{\max }\right)$, we obtain the solution

$$
u(x) \sim \begin{cases}\frac{3 \bar{u}}{2 \bar{x}^{3}}\left(\bar{x}^{2}-x^{2}\right) & 0 \leqslant x<\bar{x}=\left(\frac{9}{2} \bar{u}^{2} /|Q(\epsilon)|\right)^{1 / 4} \\ \epsilon & \bar{x}<x \leqslant 1 .\end{cases}
$$

From (43), for $\epsilon \rightarrow 0, Q(\epsilon)=\mathrm{O}\left(\epsilon^{-(n-1)}\right)$ and we obtain

$$
\bar{x} \sim \sqrt{\bar{u}} \mathrm{O}\left(\epsilon^{(n-1) / 4}\right) \rightarrow 0 \quad \bar{p} \sim \mathrm{O}\left(\epsilon^{-3(n-1) / 4}\right) / \sqrt{\bar{u}} \rightarrow \infty .
$$

Hence for $\epsilon \rightarrow 0$, equation (46) is a delta sequence, which converges to a Dirac $\delta$-distribution with mass $\bar{u}, \bar{u} \delta(x)$. As $\epsilon$ decreases, equation (46) approaches a parabolic profile with fixed area and support that vanishes as $\epsilon \rightarrow 0$. The stable solutions for a sequence of decreasing $\epsilon$ with fixed mass $\bar{u}=1$ are shown in figure 8 .

An estimate of the maximum mass $\bar{u}_{1}^{\max }$ of the first branch of the bifurcation diagram is given by the intersection of the two asymptotic families of solutions. This occurs when $u_{\max } \sim 3 \bar{u} / 2$ and (44) are set equal to yield

$$
\bar{u} \leqslant \bar{u}_{1}^{\max } \sim \frac{2}{9} \sqrt{2|Q(\epsilon)|} .
$$

Figure 5 shows that this is an excellent estimate for the position of the turning point in the $k=1$ branch. Note that the entire set of stable localized solutions has saturated pressure, $\max _{x} P(u(x))=P_{\max }$, though this condition does not define the position of the turning point (48).

Furthermore, we can make use of a result of Laugesen and Pugh [35] to validate the asymptotics. The contrapositive of theorem 5 of [38] states that if an equilibrium solution of (13) is stable then the ratio $R(u)=G(u) / F(u)$ must be non-convex over part of the range of $u(x)$. In our problem, this ratio is $R(u)=-P^{\prime}(u)$. The condition for non-convexity is that $R^{\prime \prime}(u)=-P^{\prime \prime \prime}(u)<0$. This condition holds if $u_{\min }<u_{r}$,

$$
u_{\min }<u_{r}=\left[\frac{m(m+1)(m+2)}{n(n+1)(n+2)}\right]^{1 /(m-n)} \epsilon
$$

We note that this stability criterion, $u_{r}>u_{\min }$ [38], yields a weaker upper bound than the condition for the occurrence of an interior pressure maximum, $u_{p}>u_{\min }$ since $u_{r}>u_{p}$. 
Remark. We note that the asymptotic structure of the $k$-fold symmetric solutions can be found analogously by solving for the monotonic solutions of (39) on $0 \leqslant x \leqslant 1 / k$. Alternatively, the results for the $k=1$ branch of solutions can be mapped onto the other branches $k=2, \ldots, K$ at different values of $\epsilon$ using the scalings,

$$
\hat{x}=\ell x \quad \hat{u}=\ell^{2 /(n+1)} u \quad \hat{\epsilon}=\ell^{2 /(n+1)} \epsilon
$$

where $\ell=1 / k$ describes the $k$ th branch of solutions.

\section{Discussion}

We have proved that for a large class of admissible $(n, m)$ in (4), including the interesting physical models for van der Waals rupture, there is a common structure to the equilibrium bifurcation diagram. We found that, as $\epsilon \rightarrow 0$, the bifurcation diagram develops more branches and the stable steady state approaches the $\delta$-distribution mass concentration. Finite $\epsilon>0$ provides a regularization of (12); the resulting problem has global existence for smooth solutions. In contrast, for (12) with $\epsilon=0$, classical solutions exist only up to a finite-time rupture singularity, and the problem is ill-posed after rupture.

It is interesting to compare our study with regularizations of other problems in fluid dynamics that produce finite-time singularities. In the study of classically ill-posed twodimensional vortex sheets, well-posed regularizations, involving either viscosity [56] or a desingularization of the Birkhoff-Rott kernel [34], are used to compute past finite-time curvature singularities. Moreover, whether vorticity concentrations can occur in the flow has been an active area of research $[11,21]$. Finite-time singularities also occur in Hele-Shaw flows as a consequence of topological transitions during the break-up of bubbles. The dynamics of these flows is likely to be locally well-posed before and after breakup, however, it is unclear how to pass through the topology change $[8,49]$. A diffuse interface approach provides a regularization of the Hele-Shaw problem that is globally well-posed [19,40,41]. In our study, for finite $\epsilon>0$, the conjoining pressure term in (4) regularizes the $\epsilon=0$ finite-time rupture problem. For $\epsilon>0$ the evolution is well-posed through topological transitions, as in diffusive interface models for Hele-Shaw flows. And, for $\epsilon \rightarrow 0$, we will present numerical simulations of the dynamics of (12) that show the occurrence of concentrations.

To examine the formation of mass concentrations as $\epsilon \rightarrow 0$ after rupture, we consider a sequence of numerical solutions $u^{\epsilon}(x, t)$ of the initial-value problem for (12) with $L=1$, $M(u)=u^{3}$, and $P(u)=u^{-3}-\epsilon u^{-4}$, with the monotonically decreasing initial data $u_{0}(x)=0.4+0.002 \cos (\pi x)$. Figure 9 shows the maximum and minimum of the solution as a function of time for the following values of $\epsilon: 0.1,0.05,0.025,0.0125,0.00625$, and $\epsilon=0$. The $\epsilon=0$ problem exhibits finite-time rupture at time $T_{R} \approx 0.041$. For $\epsilon>0$, the solutions do not rupture, but each 'dewets' shortly after $T_{R}$, that is the minimum of each solution becomes small, $u_{\min }(t)=\mathrm{O}(\epsilon)$. Thereafter, the $\epsilon>0$ solutions rapidly converge to the unique non-trivial stable steady-state solution for this problem (see figure 8 ). The graphs of the minimum and maximum of the solutions $u^{\epsilon}(x, t)$ shown in figure 9 give one indicator of the convergence to the steady state. We find that for smaller values of $\epsilon$ the convergence to steady state happens on shorter time scales. Since the steady states converge to a Dirac $\delta$-distribution, the computations suggest that for each time after the rupture time $T_{R}$, the time-dependent solution sequence converges to a $\delta$-distribution, i.e. for all $t \geqslant 0$,

$$
\lim _{\epsilon \rightarrow 0} u^{\epsilon}(x, t)= \begin{cases}u^{0}(x, t) & t \leqslant T_{R} \\ \bar{u} \delta(x) & t>T_{R} .\end{cases}
$$




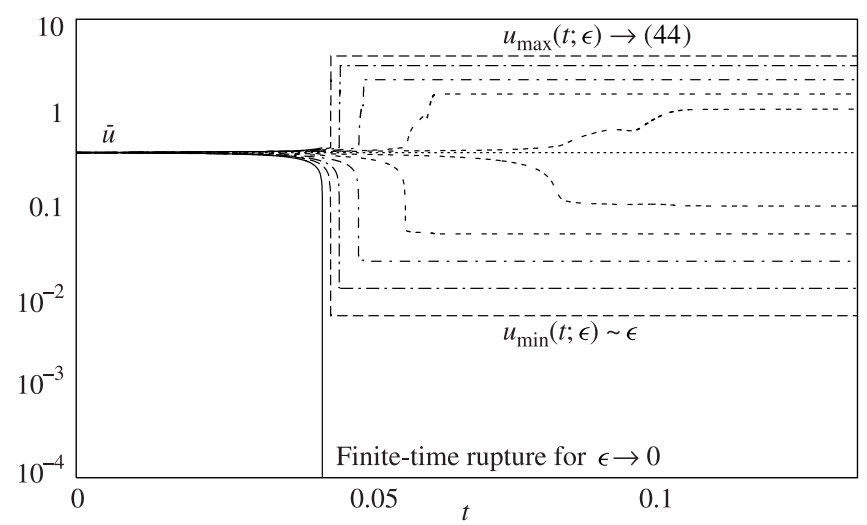

Figure 9. A sequence of dynamic simulations in the $\epsilon \rightarrow 0$ limit. Numerical results indicate convergence to the equilibrium rapidly after rupture for $\epsilon \rightarrow 0$.

Thus concentrations occur in this solution sequence for $\epsilon \rightarrow 0$. In fact, the only estimate, independent of $\epsilon$, that is known for the PDE is the $L^{1}$ norm, that is the conservation of mass. This simulation of $u^{\epsilon}(x, t)$ suggests that sharper analytical control is not possible. Finally, it is clear from this simulation that there is a droplet formation timescale, $T_{D}$, i.e. the time it takes for the solution to converge to a parabolic droplet after the onset of dewetting at $T_{R}$. This preliminary numerical study suggests that $T_{D} \rightarrow 0$ as $\epsilon \rightarrow$ 0 .

To briefly study the influence of the limit $\epsilon \rightarrow 0$ on the dynamics of topological transitions (described in [47] as 'morphological phase separation'), we look at simulations illustrating long-time coarsening dynamics in (12). We use the algorithm in [26] to compute the solution of (12) with $M(u)=u^{3}, P(u)=u^{-3}-\epsilon u^{-4}, L=20$, and the initial data $u_{0}(x)=1+0.005 \sin \left(6(x-13)^{2}\right)$, for $\epsilon=0.1$ and 0.01 . Unlike the simple monotonic initial data used in figure 9 , these new initial data have the potential to form complicated patterns of droplets that may depend sensitively on the structure of $u_{0}(x)$. As shown in figure 10, these simulations illustrate the dynamics of dewetting (or formation of 'dry spots') and coarsening starting from small perturbations of a flat thin film. Similar results have been observed in other studies for fixed values of $\epsilon[43,45,47]$; our interest here is the dependence of the dynamics on $\epsilon$, in the limit that $\epsilon \rightarrow 0$. Figure 10 shows the results for $\epsilon=0.1$ and 0.01 . In both situations, after an initial period of dewetting dynamics, the solutions converge to a series of droplets, at arbitrary positions in the domain, generally with different masses, all connected by an ultra-thin film. Each of these droplets is close to an equilibrium solution, and, in fact, they persist for very long times. However, from section 5, we know that there cannot be any stable equilibria with interior extrema [38], hence these states are unstable. These states are called metastable, and their long-time dynamics has been studied for Cahn-Hilliard equations [51,59]. The long-time dynamics is characterized by coarsening instabilities that ultimately lead to a final stable droplet (see figure 8) which is the global minimizer. The time needed for the coarsening process depends on $\epsilon$, with smaller $\epsilon$ requiring more time to evolve to the global minimizer. Thus there is a coarsening time scale, $T_{C}$, that describes the time it takes for the number of droplets in the solution to decrease. As suggested by figure 10, this time scale increases, $T_{C} \rightarrow \infty$, as $\epsilon \rightarrow 0$, a dramatically different dependence on $\epsilon$ than the droplet formation time-scale, $T_{D} \rightarrow 0$. 

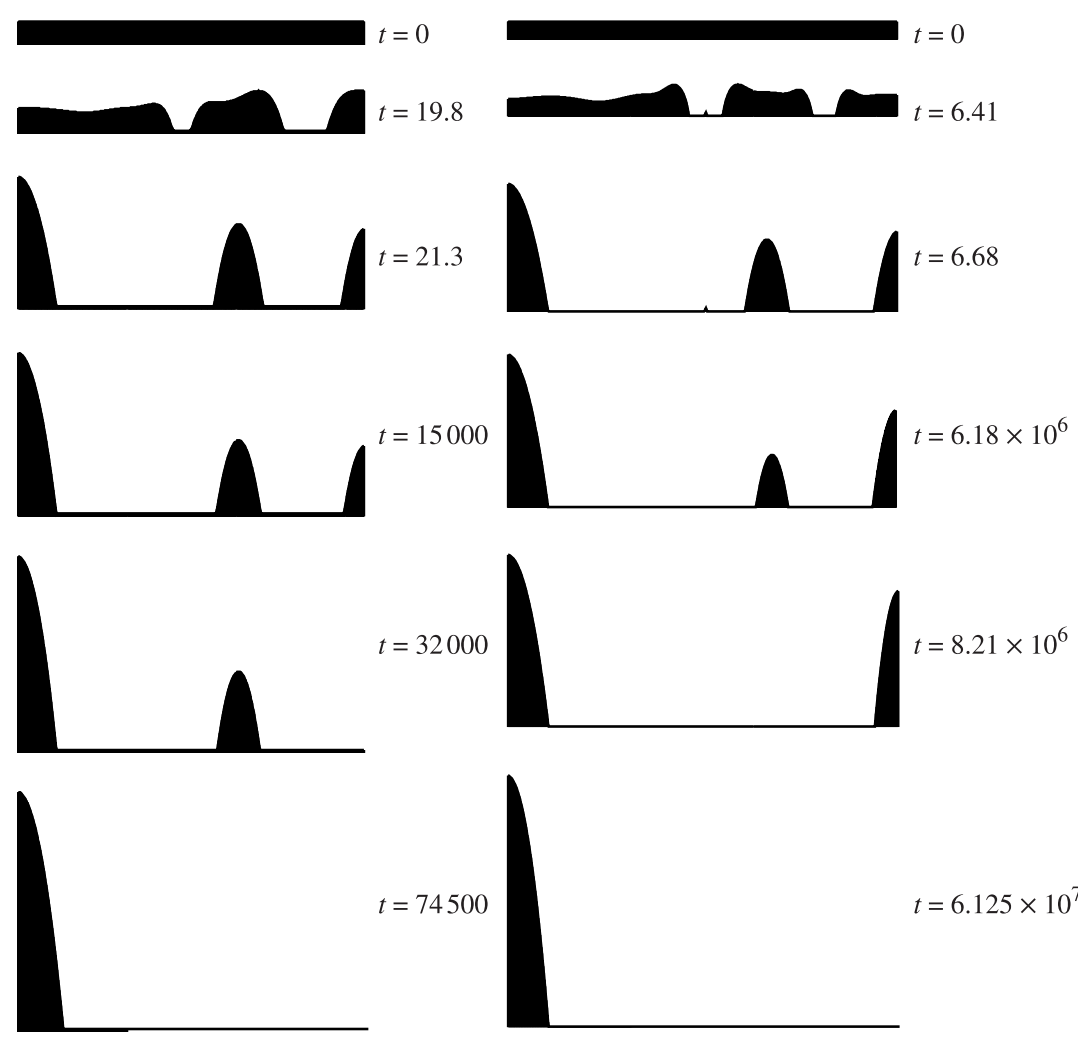

Figure 10. The long-time dynamics for (12) with $n=3, m=4$ for $\epsilon=0.1$ (left) and $\epsilon=0.01$ (right). Note that the time-scale for the coarsening process increases as $\epsilon \rightarrow 0$ and that the final state converges to the global minimizer.

Physical problems for droplet formation involve instabilities of two-dimensional thin films, $u=u(x, y, t)$. These problems have more complicated dynamics and a richer set of equilibrium patterns, given by the solutions of nonlinear elliptic equations. Moreover, analysis of some fundamental issues, such as positivity and pointwise boundedness, for the dynamics of (1)-(4) remain as open questions [25]. Specific analysis problems are raised by this study, including a proof of the small- $\epsilon$ asymptotics of the bifurcating branches and the details of the location of the turning points. Some progress has been made on related questions for the Cahn-Hilliard equation (see [22] and references therein).

We conjecture that mass concentrations for $\epsilon \rightarrow 0$ also exist for our problem in two dimensions. Of course, the one-dimensional equilibrium droplet solutions $u(x)$ we have described exist in two dimensions as fluid ridges, with parabolic cross sections. We expect that these fluid cylinders are unstable and will break up to form patterns of axisymmetric droplets. It is also interesting to study the dynamics of the coarsening process and the statistics of droplet sizes over time for general initial conditions and in comparison with experimental data [33,53-55]. It is important to study whether the physics on very small scales, which sets a finite value for $\epsilon$ and controls the time-scales in the model, can be accurately represented by a simple continuum model such as (4). 


\section{Acknowledgments}

We thank Andrew Bernoff, Richard Laugesen and Mary Pugh for useful comments. The simulation shown in figure 10 was performed by Jürgen Becker who is supported by grant Gr1693/1-1 of the priority programme Benetzung und Strukturbildung an Grenzflächen of Deutsche Forschungsgemeinschaft. GG gratefully acknowledges the hospitality of the Mathematics Department at Duke University. AB and TW are supported by ONR grant N00014-96-1-0656 and NSF FRG grant DMS-0074049. TW is also supported by a fellowship from the Alfred P Sloan Foundation.

\section{References}

[1] Bai F S, Elliott C M, Gardiner A, Spence A and Stuart A M 1995 The viscous Cahn-Hilliard equation. I. Computations Nonlinearity 8 131-60

[2] Bai F S, Spence A and Stuart A M 1994 Numerical computations of coarsening in the one-dimensional CahnHilliard model of phase separation Physica D 78 155-65

[3] Bates P W and Fife P C 1990 Spectral comparison principles for the Cahn-Hilliard and phase-field equations and time scales for coarsening Physica D 43 335-48

[4] Bernis F and Friedman A 1990 Higher order nonlinear degenerate parabolic equations J. Diff. Eq. 83 179-206

[5] Bertozzi A L and Pugh M C 1998 Long-wave instabilities and saturation in thin film equations Commun. Pure Appl. Math. 51 625-61

[6] Bischof J, Scherer D, Herminghaus S and Leiderer P 1996 Dewetting modes of thin metallic films: nucleation of holes and spinodal dewetting Phys. Rev. Lett. 77 1536-9

[7] Burelbach J P, Bankoff S G and Davis S H 1988 Nonlinear stability of evaporating/condensing liquid films $J$. Fluid Mech. 195 463-94

[8] Constantin P and Pugh M C 1993 Global solutions for small data to the Hele-Shaw problem Nonlinearity 6 393-415

[9] Dal Passo R, Garcke H and Grün G 1998 On a fourth-order degenerate parabolic equation: global entropy estimates, existence and qualitative behavior of solutions SIAM. J. Math. Anal. 29 321-42 (electronic)

[10] de Gennes P G 1985 Wetting: statics and dynamics Rev. Mod. Phys. 57 827-80

[11] DiPerna R J and Majda A J 1987 Concentrations in regularizations for 2D incompressible flow Commun. Pure Appl. Math. 40 301-45

[12] Eilbeck J C, Furter J E and Grinfeld M 1989 On a stationary state characterization of transition from spinodal decomposition to nucleation behaviour in the Cahn-Hilliard model of phase separation Phys. Lett. A 135 $272-5$

[13] Elbaum M, Lipsom S G and Wettlaufer J F 1995 Evaporation pre-empts complete wetting Europhys. Lett. 29 457-62

[14] Eres M H, Schwartz L W and Roy R V 2000 Fingering phenomena for drive coating flows Phys. Fluids 12 1278-95

[15] Erneux T and Gallez D 1997 Can repulsive forces lead to stable patterns in thin liquid films? Phys. Fluids 9 1194-6

[16] Evans L C 1990 Weak Convergence Methods for Nonlinear Partial Differential Equations (CBMS Lecture Notes 74) (Providence, RI: American Mathematical Society)

[17] Evans L C 1998 Partial Differential Equations (Providence, RI: American Mathematical Society)

[18] Giacomelli L and Otto F 2000 Variational formulation of the lubrication approximation of the Hele-Shaw flow Calculus of Variations and Partial Differential Equations to appear

[19] Glasner K 2001 A diffuse interface approach to Hele-Shaw flow Nonlinearity submitted

[20] Golubitsky M and Schaeffer D G 1985 Singularities and Groups in Bifurcation Theory vol I (New York: Springer)

[21] Greengard C and Thomann E 1988 On DiPerna-Majda concentration sets for two-dimensional incompressible flow Commun. Pure Appl. Math. 41 295-303

[22] Grinfeld M and Novick-Cohen A 1999 The viscous Cahn-Hilliard equation: Morse decomposition and structure of the global attractor Trans. Am. Math. Soc. 351 2375-406

[23] Grisvard P 1992 Singularities in Boundary Value Problems (Paris: Masson)

[24] Grün G 1995 Degenerate parabolic equations of fourth order and a plasticity model with nonlocal hardening $Z$ Anal. Anwendungen 14 541-73 
[25] Grün G 2000 On the convergence of entropy consistent numerical schemes for lubrication-type equations in multiple space dimensions submitted

[26] Grün G 2001 On the numerical simulation of wetting phenomena Proc. 15th GAMM-Seminar Kiel, Numerical Methods of Composite Materials ed W Hackbusch and S Sauter (Braunschweig: Vieweg) to appear

[27] Grün G and Rumpf M 2001 Simulation of singularities and instabilities arising in thin film flow Eur. J. Appl. Math. 12 293-320

[28] Herminghaus S, Jacobs K, Mecke K, Bischof J, Fery A, Ibn-Elhaj M and Schlagowski S 1998 Spinodal dewetting in liquid crystal and liquid metal films Science 282 916-9

[29] Holmes M H 1995 Introduction to Perturbation Methods (New York: Springer)

[30] Israelachvili J N 1992 Intermolecular and Surface Forces 2nd edn (New York: Academic)

[31] Keller J B and Antman S (ed) 1969 Bifurcation Theory and Nonlinear Eigenvalue Problems (New York: Benjamin)

[32] Kevorkian J and Cole J D 1996 Multiple Scale and Singular Perturbation Methods (New York: Springer)

[33] Konnur R, Kargupta K and Sharma A 2000 Instability and morphology of thin liquid films on chemically heterogeneous substrates Phys. Rev. Lett. 84 931-4

[34] Krasny R 1986 Desingularization of periodic vortex sheet roll-up J. Comput. Phys. 65 292-313

[35] Laugesen R S and Pugh M C 2000 Private communication

[36] Laugesen R S and Pugh M C 2001 Energy levels of steady states for thin film type equations J. Diff. Eq. to be published

[37] Laugesen R S and Pugh M C 2000 Heteroclinic orbits, mobility parameters and stability for thin film type equations Preprint AP/0003209 http://xyz.lanl.gov/abs/math

[38] Laugesen R S and Pugh M C 2000 Linear stability of steady states for thin film and Cahn-Hilliard type equations Arch. Ration. Mech. Anal. 154 3-51

[39] Laugesen R S and Pugh M C 2000 Properties of steady states for thin film equations Eur. J. Appl. Math. 11 293-351

[40] Lowengrub J and Truskinovsky L 1998 Quasi-incompressible Cahn-Hilliard fluids and topological transitions R. Soc. Lond. Proc. A 454 2617-54

[41] Lowengrub J S, Goodman J, Lee H, Longmire E K, Shelley M J and Truskinovsky L 1999 Topological transitions in liquid/liquid interfaces Free Boundary Problems: Theory and Applications (Crete, 1997) (London: Chapman and Hall/Boca Raton, FL: Chemical Rubber Company) pp 221-36

[42] Mitlin V S 1993 Dewetting of a solid surface: analogy with spinodal decomposition J. Coll. Int. Sci. 156 491-7

[43] Mitlin V S and Petviashvili N V 1994 Nonlinear dynamics of dewetting: kinetically stable structures Phys. Lett. A $192323-6$

[44] Novick-Cohen A and Peletier L A 1993 Steady states of the one-dimensional Cahn-Hilliard equation Proc. R. Soc. Edin. A 123 1071-98

[45] Oron A and Bankoff S G 1999 Dewetting of a heated surface by an evaporating liquid film under conjoining/disjoing pressures J. Coll. Int. Sci. 218 152-66

[46] Oron A and Bankoff S G 2001 Dynamics of a condensing liquid film under conjoining/disjoining pressures Phys. Fluids 13 1107-17

[47] Oron A, Davis S H and Bankoff S G 1997 Long-scale evolution of thin liquid films Rev. Mod. Phys. 69 931-80

[48] Otto F 1998 Lubrication approximation with prescribed nonzero contact angle Commun. Partial Diff. Eq. 23 2077-164

[49] Prokert G 1998 Existence results for Hele-Shaw flow driven by surface tension Eur. J. Appl. Math. 9 195-221

[50] Reiter G 1992 Dewetting of thin polymer films Phys. Rev. Lett. 68 75-8

[51] Reyna L G and Ward M J 1995 Metastable internal layer dynamics for the viscous Cahn-Hilliard equation Methods Appl. Anal. 2 285-306

[52] Schaaf R 1990 Global Solution Branches of Two-Point Boundary Value Problems (Berlin: Springer)

[53] Schwartz L W, Roy R V, Eley R R and Petrash S 2001 Dewetting patterns in a drying liquid film J. Coll. Int. Sci. 234 363-74

[54] Sharma A and Khanna R 1998 Pattern formation in unstable thin liquid films Phys. Rev. Lett. 81 3463-6

[55] Sharma A and Khanna R 1999 Pattern formation in unstable thin liquid films under the influence of antagonistic short- and long-range forces J. Chem. Phys. 110 4929-36

[56] Tryggvason G, Dahm W J A and Sbeih K 1991 Fine structure of vortex sheet rollup by viscous and inviscid simulation J. Fluids Eng. Trans. ASME 113 31-6

[57] Valignat M P, Villette S, Li J, Barberi R, Bartolino R, Dubois-Violette E and Cazabat A M 1996 Wetting and anchoring of a nematic liquid crystal on a rough surface Phys. Rev. Lett. 77 1994-7

[58] Vandenbrouck F, Valignat M P and Cazabat A M 1999 Thin nematic films: metastability and spinodal dewetting Phys. Rev. Lett. 82 2693-6 
[59] Ward M J 1994 Metastable patterns, layer collapses and coarsening for a one-dimensional Ginzburg-Landau equation Stud. Appl. Math. 91 51-93

[60] Williams M B and Davis S H 1982 Nonlinear theory of film rupture J. Coll. Int. Sci. 90 220-8

[61] Witelski T P and Bernoff A J 1999 Stability of self-similar solutions for van der Waals driven thin film rupture Phys. Fluids 11 2443-5

[62] Witelski T P and Bernoff A J 2000 Dynamics of three-dimensional thin film rupture Physica D 147 155-76

[63] Xie R, Karim A, Douglas J F, Han C C and Weiss R A 1998 Spinodal dewetting of thin polymer films Phys. Rev. Lett. 81 1251-4

[64] Zhang W W and Lister J R 1999 Similarity solutions for van der Waals rupture of a thin film on a solid substrate Phys. Fluids 11 2454-62 